\title{
EFFECT OF A VARIABLE DELAY IN DELAYED DYNAMICAL SYSTEMS
}

\author{
S. MADRUGA and S. BOCCALETTI
}

\author{
M. A. MATÍAS
}

\begin{abstract}
We report numerical evidence of the effects of a periodic modulation in the delay time of a delayed dynamical system. By referring to a Mackey-Glass equation and by adding a modulation in the delay time, we describe how the solution of the system passes from being chaotic to shadow periodic states. We analyze this transition for both sinusoidal and sawtooth wave modulations, and we give, in the latter case, the relationship between the period of the shadowed orbit and the amplitude of the modulation. Future goals and open questions are highlighted.
\end{abstract}

In nature a nonzero delay time occurs always between the instants at which a cause and its effects take place. In some situations, such a delay comes out to be negligible with respect to the timescales of the dynamics and therefore the usual ordinary differential equation model well represents the natural behavior. However, there are relevant cases in which finite propagation speeds of signals, transport effects, finite reaction times, or finite response times of the system must be taken into account. As a result, the most suitable model equations to describe these cases are the so-called delayed dynamical system (DS). DS are equations of the form

$$
\dot{x}=\mathcal{F}\left(x, x_{d}, \lambda\right),
$$

where $x$ is a $m$-dimensional vector $(m \geq 1)$ representing the order parameter of the system, dot denotes temporal derivative, $\mathcal{F}$ is a generic nonlinear function, $\lambda$ is a family of control parameters, $x_{d} \equiv x(t-\tau)$, and $\tau$ is the delay time.

Because of their infinite dimensionality, DS provides a natural link between concentrated and space extended systems (ES). The analogy between DS and ES was first given experimentally for a $\mathrm{CO}_{2}$ laser with delayed feedback [Arecchi et al., 1992], and then supported by a theoretical model [Giacomelli \& Politi, 1996]. The DS to ES conversion is based on the following two variable representations of the time

$$
t=\sigma+\theta \tau,
$$

where $0 \leq \sigma \leq \tau$ is a continuous space-like variable and $\theta$ is a discrete temporal variable [Arecchi et al., 1992]. By means of Eq. (2) the long range interactions caused by the delay are converted into short range interactions along the direction $\theta$, since $x_{d} \equiv x(\sigma, \theta-1)$. In this framework, the formation and propagation of space-time structures, such as defects [Giacomelli et al., 1994] and/or spatiotemporal intermittency, have been identified [Giacomelli \& Politi, 1996] and controlled [Boccaletti et al., 1997].

There are aspects which confirm such analogy. One example is the growth of the number of positive 
Lyapunov exponents with the delay $\tau$ for DS, which is linear similar to what happens in one-dimensional ES with the size of the system in the regime of weak space-time chaos [Pomeau et al., 1984]. A linear relation between delay and attractor dimensions was found by Farmer [1982] for the MackeyGlass system [Mackey \& Glass, 1977] and the Ikeda map [Ikeda \& Matsumoto, 1986], thus indicating that increasing the delay time has the same effect as increasing the size of a one-dimensional extended system. There are aspects, however, for which the analogy between DS and ES fails. An example is the metric entropy, which is constant as a function of the delay time for DS in a chaotic regime, whereas in general it can vary as a function of the system extension for ES.

The increasing attention that the scientific community devoted to DS is witnessed by the huge number of DS proposed in the literature to model a variety of different systems. In biophysics, the Mackey-Glass model has been introduced to describe the process of blood cells creation [Mackey \& Glass, 1977]. Other examples of DS are the logistic equation modeling the population evolution [May, 1976], or the Ikeda equation [Ikeda, 1979] modeling the evolution of the light in a ring cavity, or the Lang-Kobayashi model [Lang \& Kobayashi, 1980] describing a semiconductor laser in the single-mode regime with delayed optical feedback.

In this Letter, we will perform a step forward by taking into account the effects of a variable delay on the dynamical behavior of a system, i.e. considering $\tau=\tau(t)$. We argue that this provides an even more realistic viewpoint, insofar as the existence of variable delays is a ubiquitous phenomenon in nature. As an example, one can refer to the influence of stochastic processes over transport speeds in chemical reactions or electronic noise through a communication channel, leading to $x_{d}=x(t-\tau+\xi)$, $\xi$ being a white noise term with zero mean and $\delta$ correlation in time. Besides stochastic processes, one can have other types of variable delays, such as periodic delays $\left(x_{d}=x(t-\tau+f(t)), f\right.$ being a periodic function. This latter case is present, e.g. in external forcings where the amplitude of the force may determine the delay effects. One example is biological biorhythms: the capacity of assimilation of nutrients by an organism varies cyclicly during the day.

As one can expect, a variable delay may crucially change the dynamics of a DS. A recent work has pointed out that a very tiny change in time of the delay can even control a high dimensional chaotic DS giving rise to simple periodic behavior [Maza et al., 1998].

Furthermore, the fractal dimensions for a DS increase linearly with the delay [Farmer, 1982], therefore the robustness of high dimensional chaotic DS (DS with large delays) against modulations in the delay time is an interesting issue.

We address numerically the effects of a periodic modulation in the delay time for the Mackey-Glass model, focussing on the strong changes induced by this modulation on the dynamics.

The system under study is

$$
\dot{x}=-0.1 x(t)+0.2 \frac{x(t-\tau(t))}{1+x(t-\tau(t))^{10}},
$$

where $x(t)$ is the concentration of mature cells in blood at time $t$, and $\tau(t)$ is the delay between the initiation of cellular production in the bone marrow and the release of mature cells into the blood. The above model equation represents a new kind of dynamical systems, that we will call from now on delayed dynamical systems with variable delay, or variable delayed dynamical systems (VDS).

The case of constant delay $\left(\tau(t)=\tau_{0}\right)$ has been extensively studied in literature [Mackey \& Glass, 1977; Farmer, 1982], and has been taken as a prototypic example for testing the robustness of control [Boccaletti et al., 1997; Maza et al., 1998] and synchronization [Pyragas, 1998; Bünner \& Just, 1998; Boccaletti et al., 2000] techniques over high dimensional chaotic states. Linear stability analysis of Eq. (3) shows that $x=1$ is a stable fixed point for $\tau \leq 4.7$. Numerically, one observes a stable limit cycle of period one for $4.7 \leq \tau \leq 13.3$. At $\tau=13.3$, a period doubling bifurcation cascade starts until $\tau=16.8$, where a chaotic behavior sets in, intermingled with some periodic windows.

For the time being, we consider two different kinds of periodic modulation, namely a sinusoidal one of the form

$$
\tau(t)=\tau_{0}+A \sin (\omega t),
$$

$\tau_{0}$ being the zero frequency component, $A \leq \tau_{0}$ the amplitude and $\omega / 2 \pi$ the frequency of the modulation, and a periodic sawtooth wave of the form

$$
\tau(t)=\tau_{0}+\tau_{0}(\nu t-\operatorname{Int}(\nu t))
$$

$\tau_{0}$ being the zero frequency component, $\nu$ the frequency of the modulation and $\operatorname{Int}(\nu t)$ being the integer part of $\nu t$. In this latter case, the amplitude 
of the modulation coincides with $\tau_{0}$, and therefore the value of the delay time is ranging in the interval $\tau_{0} \leq \tau(t) \leq 2 \tau_{0}$. Furthermore, the modulation considered in Eq. (5) is not continuous at all times $t_{n}=n / \nu, n=1,2, \ldots$.

For a DS, when analytical treatment is possible, the search for solutions can be performed with two techniques, namely the integration by time intervals in which the value of $x$ in a given time interval of length $\tau$ is used to update the DS in the next time interval of length $\tau$, and the Laplace transform, allowing to convert a DS into a set of algebraic transcendental equations, thus being very useful for the linear stability analysis. However, as one can expect, very few situations call for analytical solutions, and often one has to rely on numerical integration schemes.

One possible approach consists in expanding the delayed variables $x_{d}=x(t-\tau)$ in Taylor series around $t_{0}\left(x\left(t_{0}-\tau\right)=x\left(t_{0}\right)-\left.\tau(d x / d t)\right|_{t_{0}}+\right.$ $\left.\left.\left(\tau^{2} / 2\right)\left(d^{2} x / d x^{2}\right)\right|_{t_{0}}+\cdots\right)$. However, this technique is subjected to some drawback, and in some cases it does not work correctly [MacDonald, 1989]. Therefore, a more robust technique comes out to be adapting a given numerical method for ordinary differential equations to integrate the particular DS under study. The most simple algorithm for fixed delay consists in storing an array of $x$ values in the interval $[t-\tau, t]$ to integrate the equations at time $t$. A new strategy has been recently introduced [Baker et al., 1994], which makes use of a fifthorder Hermite interpolant together with an embedded Runge-Kutta [Dormand \& Pierce, 1980] and a tracking process of discontinuities (for details we address the reader to [Baker et al., 1994]). In the following we will extend this latter strategy to numerically integrate our VDS for the two different choices of delay modulation.

Let us begin by reporting the results of the numerical integrations of Eq. (3) with a sinusoisal modulation in the delay, for different values of $\tau_{0}$, $A$ and $\omega$. In the considered trial, $\tau_{0}>16.8$ (the onset of chaotic dynamics at fixed $\tau$ ). For each $\tau_{0}$, three different values of $A$ have been considered, namely $A=\tau_{0} / 1000, A=\tau_{0} / 100$ and $A=\tau_{0} / 10$. The above choices implies that in all integrations the system is considered inside the regime which would be chaotic at fixed delay time, i.e. we move our parameter in a region where similar values of a fixed delay would induce high dimensional chaotic dynamics.
For the majority of chosen modulation parameters, the solutions are chaotic. However, some islands of parameters exist where the system shows a periodic behavior. Looking at Fig. 1 one can appreciate the effect of the modulation in the delay time, inducing a passage of the system from a high dimensional chaotic state of dimension $D \simeq 5$ [Farmer, 1982] to a periodic state. For other choices of parameters, the chaotic attractor collapses into periodic orbits of different periods.

From a general point of view, this collapse of the chaotic attractor into a periodic one occurs only for very particular choices of the parameters. Namely, we have not encountered collapses phenomena for amplitudes of the modulation lower than $10 \% \tau_{0}$, and therefore we have concentrated our efforts to produce ordered evolutions for perturbations larger than the above threshold. As for the frequency of the modulation, as one can expect, very low frequencies are not effective in changing the chaotic dynamics (which, indeed, would correspond to $\omega=0$ ), and one has to force with a minimum characteristic modulation time to induce an ordered (periodic) solution.

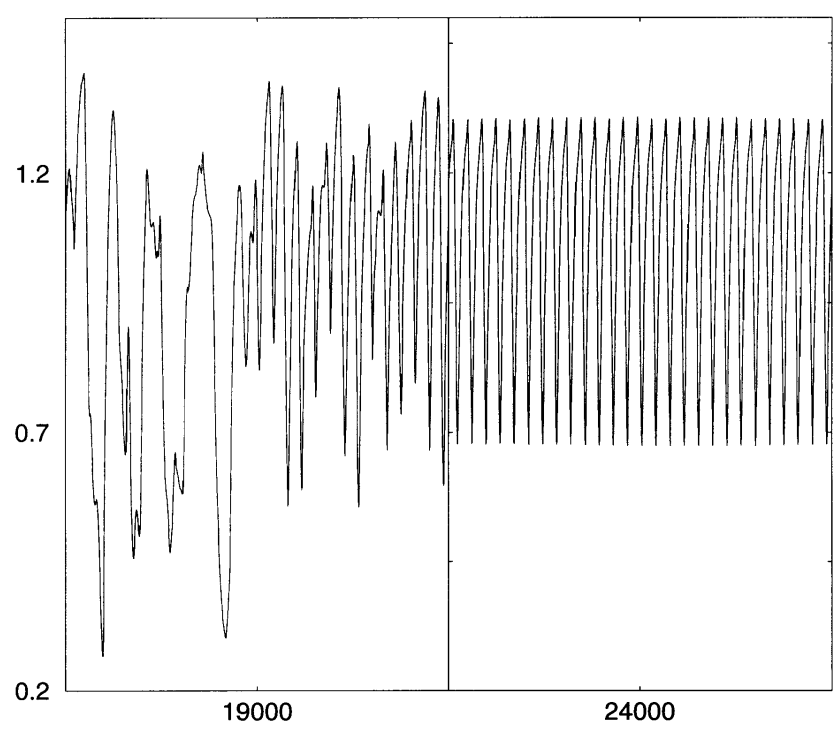

Fig. 1. Numerical simulation of Eq. (3) with a sinusoidal modulation in the delay time. Horizontal axis reports time in a.u., vertical axis reports the variable $x$ in a.u. The separation between tics in the horizontal axis corresponds to 500 time units. The modulation is switched on at time $t=15000$. Other parameters: $\tau_{0}=50, A=8, \omega / 2 \pi=0.027$. For this choice of parameters, the system experiences a transition from a high dimensional chaotic behavior to a periodic orbit of period 1 (left part of the plot) which is eventually set in the system (right part of the plot). 
A much richer scenario is induced by the modulation of Eq. (5). In this case, the parameter space reduces to two dimensions, and numerical simulations have been performed for the same range of $\tau_{0}$ and $\nu=\omega / 2 \pi$ as above.

Our numerics indicate that, for all chosen $\tau_{0}>$ 20 and for sufficiently high modulation frequency, the system always gives rise to a solution that shadows around a periodic behavior. This is reported in Fig. 2, where one can observe the appearance of a periodic orbit that envelopes the solution as the result of the application of the modulation in the delay time. We point out that Eq. (3) is always considered inside regions where its dynamics at fixed delay time would be confined within a high dimensional chaotic attractor. As an example, in the range $50 \leq \tau(t) \leq 100\left(\tau_{0}=50\right)$, the linear relation found by Farmer at fixed delay time would imply a dimensionality $5 \leq D \leq 10$ [Farmer, 1982], whereas the system (3) always evolves around a regular periodic dynamics for suitable choices of $\nu$.

Furthermore, when the modulation (5) is active over the delay time, the transition from a chaotic attractor to the enveloping regular state is smooth as a function of the modulation frequency $\nu$. This may be better appreciated looking at the Fourier spectra of Fig. 2 for fixed $\tau_{0}$ and at different frequencies of modulation, and noticing that the effect of increasing $\nu$ results in new frequencies being destroyed in the dynamics until leading to a situation where only few Fourier components survive in the spectrum.

From what have been said above we can speak of a universal periodic orbit enveloping the solution of our VDS, induced by a triangular modulation in

$$
\begin{array}{lll}
\tau_{0}=50 & \tau_{0}=100 & \tau_{0}=150
\end{array}
$$
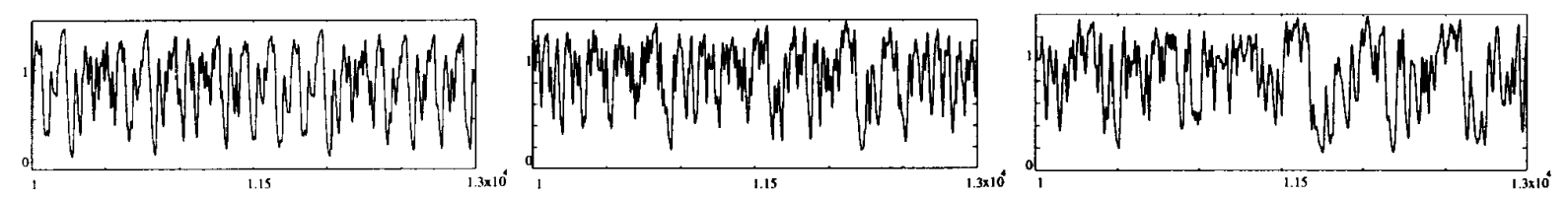

$v=0$
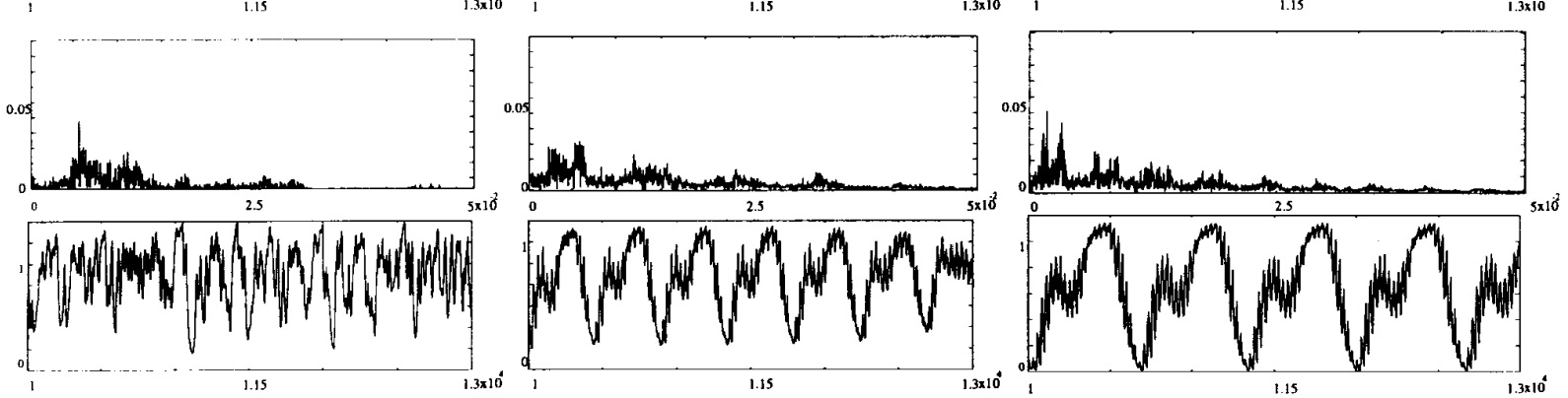

$v=0.04$
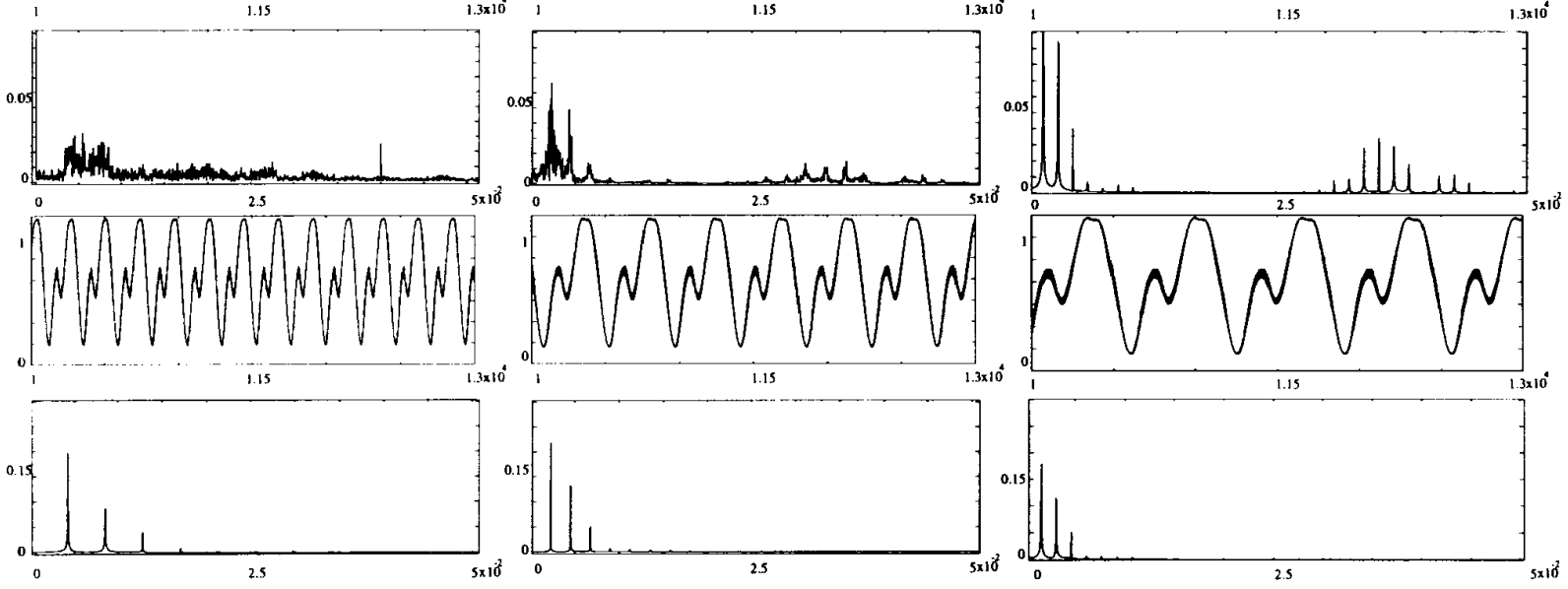

$v=0.2$

Fig. 2. Temporal evolution of the solution of Eq. (3) (first, third and fifth rows) and corresponding power spectra (second, fourth and sixth rows) for a modulation in the delay with a sawtooth wave. $\tau_{0}=50$ (first column), $\tau_{0}=100$ (second column) and $\tau_{0}=150$ (third column). The frequency of the modulation is chosen to be $\nu=0$ (first and second rows), $\nu=0.04$ (third and fourth rows) and $\nu=0.2$ (fifth and sixth rows). Notice the appearance of an enveloping limit cycle for sufficiently high forcing frequency. 
the delay time. It is worth highlighting that in this case the enveloping cycle is a period two periodic orbit, while other periods can be induced by different types of sawtooth waves.

Another feature of the observed behavior is that the greater is $\tau_{0}$ the faster the appearance of the enveloping periodic orbit as a function of $\nu$, thus indicating that very high dimensional attractors are more sensitive to variations in the delay time.

Let us move now to address how the frequency of the shadowed periodic orbit depends upon $\tau_{0}$. This is shown in Fig. 3, where the frequency of the enveloping orbit $F$ is reported to be a monotonically decreasing function of $\tau_{0}$, fitted by $F\left(\tau_{0}\right)=a+b / \tau_{0}^{c}$ $\left(a=-10^{-4}, b=0.139\right.$ and $\left.c=0.88\right)$ in the whole considered range of parameters $\left(20 \leq \tau_{0} \leq 1000\right)$. Similar curves have been found for other periodic cycles emerging with different sawtooth modulations. A direct consequence is that one can choose the frequency of the envelope of the solution by just properly tuning the amplitude of the modulation.

We believe these results could have applications in many fields such as electronics, e.g. for converting between different signal shapes; control of remote systems, laser physics or biophysics, where a modulation of the delay time can be induced to yield a desirable periodic behavior in a relatively complex dynamical system.

In conclusion, we have reported numerical evidence of the effects of a periodic variable delay time in the Mackey-Glass equation. The obtained results open several questions, such as how to characterize more quantitatively the transition to ordered states,

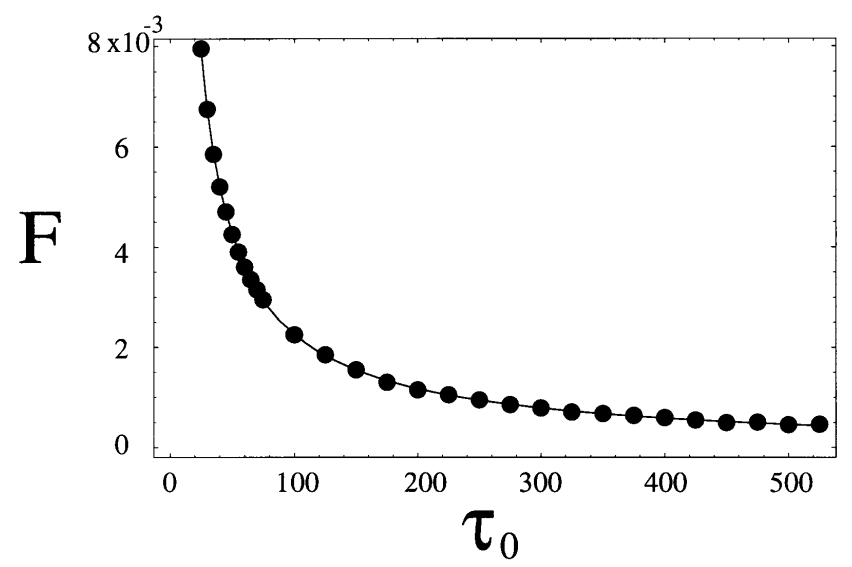

Fig. 3. Frequency of the enveloping limit cycle versus amplitude of the modulation $\tau_{0}$. Dots: numerical results; continuous line: estimating function $F\left(\tau_{0}\right)=a+b / \tau_{0}^{c}$ for $a=-10^{-4}$, $b=0.139$ and $c=0.88$. and how to build up a suitable analytical approach for VDS (similarly to what have been done for DS in [Giacomelli \& Politi, 1996]) in order to assess how feasible is their analogy with space-extended systems.

\section{Acknowledgments}

The authors acknowledge J. Kurths, I. Procaccia and H. L. Mancini for useful discussions. S. Madruga acknowledges financial support from the association "Amigos Universidad de Navarra". S. Boccaletti acknowledges financial support from EU Contract n. ERBFMBICT983466.

\section{References}

Arecchi, F. T., Giacomelli, G., Lapucci, A. \& Meucci, R. [1992] "Two-dimensional representation of a delayed dynamical system," Phys. Rev. A45, R4225-R4228.

Baker, C. T. H., Paul, C. A. H. \& Willé, D. R. [1994] Issues in the Numerical Solution of Evolutionary Delay Differential Equations, Numerical Analysis Report 248, University of Manchester.

Boccaletti, S., Maza, D., Mancini, H., Genesio, R. \& Arecchi, F. T. [1997] "Control of defects and spacelike structures in delayed dynamical systems," Phys. Rev. Lett. 79, 5246-5249.

Boccaletti, S., Valladares, D. L., Kurths, J., Maza, D. \& Mancini, H. [2000] "Synchronization of chaotic structurally nonequivalent systems," Phys. Rev. E61, $3712-3715$.

Bünner, M. J. \& Just, W. [1998] "Synchronization of time-delay systems," Phys. Rev. E58, R4072-R4075.

Dormand, J. R. \& Pierce, P. J. [1980] "A family of embedded Runge-Kutta formulae," Phil. J. Comput. Appl. Math. 6, 19-26.

Farmer, J. D. [1982] "Chaotic attractors of an infinite-dimensional dynamical system," Physica D4, 366-393.

Giacomelli, G., Meucci, R., Politi, A. \& Arecchi, F. T. [1994] "Defects and spacelike properties of delayed dynamical systems," Phys. Rev. Lett. 73, 1099-1102.

Giacomelli, G. \& Politi, A. [1996] "Relationship between delayed and spatially extended dynamical systems," Phys. Rev. Lett. 76, 2686-2689.

Ikeda, K. [1979] "Multiple-valued stationary state and its instability of the transmitted light by a ring cavity system," Opt. Commun. 30, 257-261.

Ikeda, K. \& Matsumoto, M. [1986] "Study of a highdimensional chaotic attractor," J. Stat. Phys. 44, 955-983.

Lang, R. \& Kobayashi, K. [1980] "External optical 
feedback effects on semiconductor injection laser properties," IEEE J. Quant. Electron. 16, 347-355.

MacDonald, N. [1989] Biological Delay Systems: Linear Stability Theory (Cambridge University Press, Cambridge).

Mackey, M. C. \& Glass, L. [1977] "Oscillation and chaos in physiological control systems," Science $\mathbf{1 9 7}$, 287-289.

May, R. M. [1976] "Simple mathematical models with very complicated dynamics," Nature 261, 459-467.
Maza, D., Mancini, H., Boccaletti, S., Genesio, R. \& Arecchi, F. T. [1998] "Control of amplitude turbulence in delayed dynamical systems," Int. J. Bifurcation and Chaos 8, 1843-1848.

Pomeau, Y., Pumir, A. \& Pelce, P. [1984] "Intrinsic stochasticity with many degrees of freedom," J. Stat. Phys. 37, 39-49.

Pyragas, K. [1998] "Synchronization of coupled timedelay systems: Analytical estimations," Phys. Rev. E58, 3067-3071. 